\title{
CATACLYSMIC VARIABLES AND GAMMA-RAY SOURCES
}

\author{
E. Pavlenko ${ }^{1}$, V. Malanushenko ${ }^{2}$, S. Shugarov ${ }^{3,4}$ and D. Chochol $^{4}$
}

\begin{abstract}
The cataclysmic variables are associated with high energy events and probably many of them could be potential gamma-ray sources. Up to now Fermi-LAT detected 3 gamma-ray transients, which belong to CVs and related objects: V407 Cyg, N Sco 2012 and N Mon 2012 = V959 Mon. We present the first multicolour observations of the slow classical nova V959 Mon.
\end{abstract}

\section{Introduction}

According to the modern X-ray observations, cataclysmic variables (CVs) represent a significant part of baryon matter of our galaxy (Revnivtsev et al. 2008; Pretorius \& Knigge 2012). They are the close binary stars at the late stage of evolution, formed on a time scale 0.1 - 10 Gyrs (Townsley \& Bildsten 2007). CVs are associated with high energy events. Many of CVs could be potential gammaray sources. It is worth to note that the classical Nova Cyg $1975=$ V1500 Cyg explosion disrupted spin-orbital synchronizm (Schmidt \& Stockman 1991). A nova explosion probably caused also the asynchronizm of the polar BY Cam, that currently is synchronizing with the same rate (Pavlenko et al. 2012) as V1500 Cyg (Schmidt \& Stockman 1991; Pavlenko \& Pelt 1991; Pavlenko \& Malanushenko 1996; Pavlenko \& Shugarov 2005). However only three gamma-ray transients that belong to CVs and related objects are known up to now: the symbiotic Mira V407 Cyg and classical novae N Sco 2012 and N Mon 2012, detected by Fermi-LAT.

\section{Nova Mon 2012 = V959 Mon}

Nova Monocerotis was discovered in the optical on August, 9 by S. Fujikawa (2012). It was found to be consistent with the gamma-ray transient Fermi J0639 discovered

\footnotetext{
1 Crimean Astrophysical Observatory, Crimea, Ukraine

2 Apache Point Observatory, New Mexico State University, USA

3 Sternberg Astronomical Institute, Lomonosov Moscow University, Russia

4 Astronomical Institute of the Slovak Academy of Sciences, Slovakia
} 
in early June 2012 (Cheung et al. 2012a; Cheung et al. 2012b) and XRay/UV (Nelson et al. 2012). The observations at radio were also reported by Chomiuk et al. (2012). High resolution spectroscopy and BVRI photometry for August 16-20 was presented by Munari et al. (2012). The likely progenitor of Nova was found by Greimel et al. (2012).

We started our optical observations of V959 Mon 17 days after its optical discovery. The CCD multicolour $U B V R_{J} R_{C} I_{C}, H \alpha$ observations have been carried out and are currently ongoing with the Apache Point Observatory 0.5-m telescope which is owned and operated by the Astrophysical Research Consortium and with the 0.6-m telescope of the Astronomical Institute SAS at the Stará Lesná Observatory. Here we present the behaviour of this Nova during 18 nights between August 26 and October 20 in the $U B V R_{J} R_{C} I_{C}$ bands.

The measurements of the nova were done relatively to the comparison star 2 (designated by AAVSO). For this star we obtained $U=12.13, B=12.20$, $V=11.72, R_{C}=11.48, I_{C}=11.18, R_{J}=11.36, I_{J}=11.04$.

As shown in Figure 1, the light curves of the nova V959 Mon in the $U B V R_{J} R_{C} I_{C}$ bands were different. The highest decline rate of $0.082 \mathrm{mag} / \mathrm{day}$ was detected in the $R$ band up to JD 2456201.

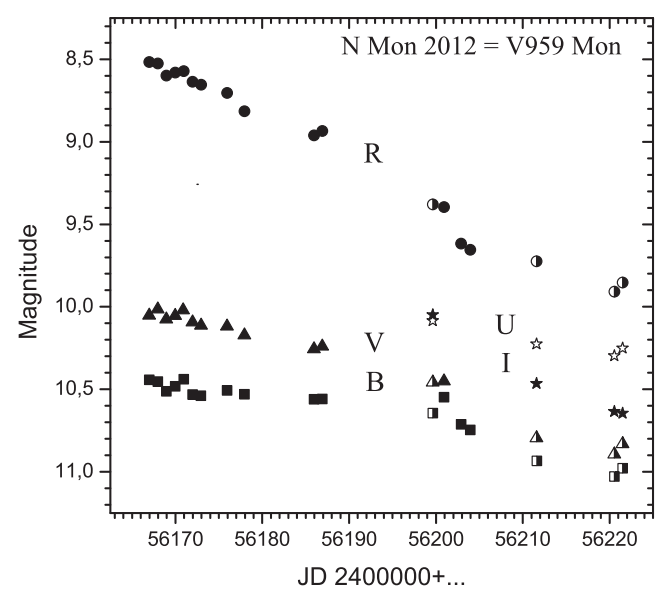

Fig. 1. The mean light curve of V959 Mon. The $U$ data are marked by empty stars (Stará Lesná), the $B$ data by filled squares (APO) and half-filled squares (Stará Lesná); the $V$ data by filled triangles (APO) and half-filled triangles (Stará Lesná); the $R_{J}$ data by filled circles (APO), the $R_{C}$ data by half-filled circles (Stará Lesná) and the $I_{C}$ data by filled stars (Stará Lesná).

As seen in two-colour diagrams presented in Figure 2, the nova V959 Mon became bluer during the decline. The $B-V, V-R_{J}$ diagram clearly show the existence of the red colour excess, probably caused by the existence of the circumstellar dust. 

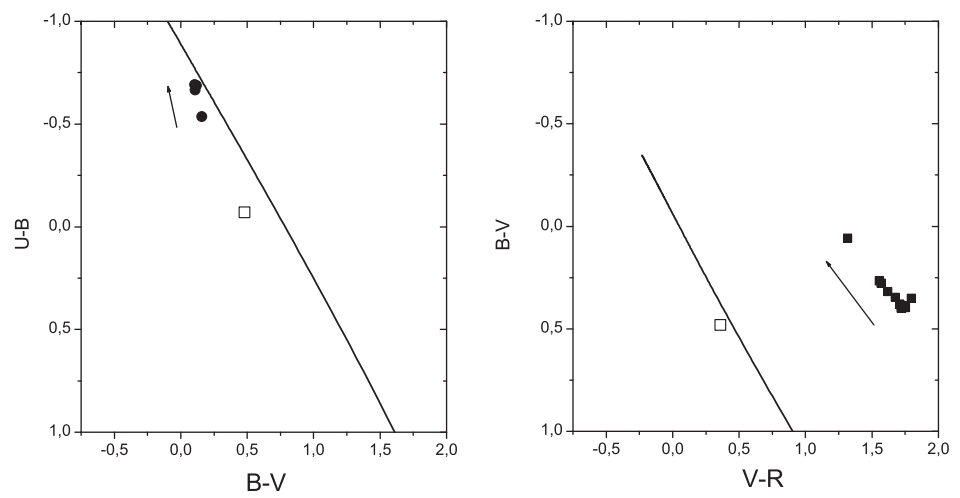

Fig. 2. The tracks of the V959 and position of the comparison star 2 (square) at the $U-B, B-V$ (for JD 2456199-2456221) and $B-V, V-R_{J}$ (for JD 2456166-2456200) diagrams. The black body in each diagram is drawn by the line. The arrow shows the change of colours of V959 Mon in chronological order.

The first observations of V959 Mon idicate that this is a very slow nova. The continuous multiwavelength observations of this unique object are strongly encouraged.

E. Pavlenko is gratefull to SOC for the financial support for participation in the Symposium. This work was partially supported by the SAIA Scholarship, program "Kosmomikrofizika" of the Ukrainian Academy of Science and the Slovak Academy of Sciences grant VEGA No. 2/0002/13. This article was supported by the realization of the Project ITMS No. 26220120029, based on the supporting operational Research and development program financed from the European Regional Development Fund.

\section{References}

Cheung, C.C., Hays, E., Venters, T., et al., 2012, ATel, 4224

Cheung, C.C., Shore, S.N., Gennaro, A., et al., 2012, ATel, 4310

Chomiuk, L., Cheung, T., Nelson, T., et al., 2012, Atel, 4352

Fujikawa, S., 2012, CBET, 3202

Greimel, R., Drew, J., Steeghs, D., et al., 2012, ATel, 4365

Munari, U., Dallaporta, S., Valisa, P., 2012, Atel, 4320

Nelson, T., Mukai, K., Chormiuk, L., et al., 2012, Atel, 4321

Pavlenko, E.P., Andreev, M., Babina, Yu., et al., 2013, ASP Conf. Ser., in press

Pavlenko, E.P., \& Pelt, Ja., 1991, Ap, 34, 169

Pavlenko, E.P., \& Malanushenko, V.P., 1996, Afz, 39, 103

Pavlenko, E.P., \& Shugarov, S.Yu., 2005, ASP Conf. Ser., 330, 421

Pretorius, M.L., \& Knigge, C., 2012 [ArXiv:1109.3162]

Revnivtsev, M., Churazov, E., Sazonov, S., et al., 2008, A\&A, 490, 37

Schmidt, G.D., \& Stockman, H.S., 1991, ApJ, 371, 749

Townsley, D.W., \& Bildsten, L., 2007, ASP Conf. Ser., 372, 557 
